\title{
Inverse Scattering Problem for the Schrödinger's Equation
}

\author{
Asset A. Durmagambetov, Leyla S. Fazilova \\ Buketov Karaganda State University, Karaganda, Kazakhstan \\ Email: aset.durmagambet@gmail.com
}

Received November 11, 2013; revised December 11, 2013; accepted December 18, 2013

Copyright (C) 2013 Asset A. Durmagambetov, Leyla S. Fazilova. This is an open access article distributed under the Creative Commons Attribution License, which permits unrestricted use, distribution, and reproduction in any medium, provided the original work is properly cited.

\section{ABSTRACT}

The analytic properties of the scattering amplitude are discussed. And, the representation of the potential by the scattering amplitude is obtained.

Keywords: Schrödinger’s Equation; Potential; Scattering Amplitude

\section{Introduction}

We consider that the operators $H=-\Delta_{x}+q(x), H_{0}=-\Delta_{x}$ are defined in the dense set $W_{2}^{2}\left(R^{3}\right)$ in the space $L_{2}\left(R^{3}\right)$ and that $q$ is a bounded fast-decreasing function. The operator $H$ is called Schrödinger's operator.

We consider the three-dimensional inverse scattering problem for the Schrödinger's operator: the scattering potential has to be reconstructed from scattering amplitude. This problem has been studied by a number of researchers (in [1-3] and references therein).

\section{Results}

We consider Schrödinger's equation:

$$
-\Delta_{x} \Psi+q \Psi=|k|^{2} \Psi, \quad k \in C
$$

Let $\Psi_{+}(k, \theta, x)$ is a solution of the (1) with the following asympotic behavior:

$$
\Psi_{+}(k, \theta, x)=\mathrm{e}^{i k \theta x}+\frac{\mathrm{e}^{i|k||x|}}{|x|} A\left(k, \theta^{\prime}, \theta\right)+0\left(\frac{1}{|x|}\right),|x| \rightarrow \infty
$$

where $A\left(k, \theta^{\prime}, \theta\right)$ scattering amplitude, and

$$
\begin{aligned}
& \theta^{\prime}=\frac{x}{|x|}, \theta \in S^{2}, \text { for } k \in \bar{C}^{+}=\{\operatorname{Im} k \geq 0\} \\
& \quad A\left(k, \theta^{\prime}, \theta\right)=-\frac{1}{4 \pi} \int_{R^{3}} q(x) \Psi_{+}(k, \theta, x) \mathrm{e}^{-i k \theta^{\prime} x} \mathrm{~d} x .
\end{aligned}
$$

We also define the solution $\Psi_{-}(k, \theta, x)$, for $k \in \bar{C}^{-}=\{\operatorname{Im} k \leq 0\}$ as $\Psi_{-}(k, \theta, x)=\Psi_{+}(-k,-\theta, x)$.

As well known [1]:

$$
\begin{aligned}
& \Psi_{+}(k, \theta, x)-\Psi_{-}(k, \theta, x) \\
& =-\frac{k}{4 \pi} \int_{S^{2}} A\left(k, \theta^{\prime}, \theta\right) \Psi_{-}\left(k, \theta^{\prime}, x\right) \mathrm{d} \theta^{\prime}, k \in R
\end{aligned}
$$

This equation is the key to solving the inverse scattering problem, and was first used by R. G. Newton in [2,3] and E. Somersalo et al. in [4].

Equation (4) is equivalent to the following:

$$
\Psi_{+}=S \Psi_{-},
$$

where $S$ is a scattering operator with the kernel $S(k, l), \quad S(k, l)=\int_{R^{3}} \Psi_{+}(k, x) \Psi_{+}^{*}(l, x) \mathrm{d} x$.

Here is a theorem according to [1]:

Theorem 1 (The energy and momentum conservation laws)

Let $q \in \mathbf{R}$, then $S S^{*}=I, \quad S^{*} S=I$, where $I$ is a unitary operator.

Definition 1 The set of measurable functions $\mathbf{R}$ with the norm, defined as $\|q\|_{\mathbf{R}}=\int_{R^{6}} \frac{q(x) q(y)}{|x-y|^{2}} \mathrm{~d} x \mathrm{~d} y<\infty$ is recognized as Rollnik' class.

As shown in [5], $\Psi_{ \pm}(k, x)$ is an orthonormal system of $H$ eigenfunctions for the continuous spectrum. In addition to the continuous spectrum there are a finite number $N$ of $H$ negative eigenvalues, designated as $-E_{j}^{2}$ with corresponding normalized eigenfunctions $\psi_{j}\left(x,-E_{j}^{2}\right)(j=\overline{1, N})$, where $\psi_{j}\left(x,-E_{j}^{2}\right) \in L_{2}\left(R^{3}\right)$. 
We present Povzner's results [5] below:

Theorem 2 (Completness) Both for arbitrary $f \in L_{2}\left(R^{3}\right)$ and for $H$ eigenfunctions Parseval's identity is valid.

$$
\begin{aligned}
|f|_{L_{2}}^{2} & =\left(P_{D} f, P_{D} f\right)+\int_{R^{3}}|\bar{f}(s)|^{2} \mathrm{~d} s \\
P_{D} f & =\sum_{j=1}^{N} f_{j} \psi_{j}\left(x,-E_{j}\right),
\end{aligned}
$$

where $f_{j}$ and $\bar{f}$ are Fourier coefficients for continuous and dicrete cases.

Theorem 3 (Birmann-Schwinger estimation). Let $q \in R$. Then number of discrete eigenvalues can be estimated as:

$$
N(q) \leq \frac{1}{(4 \pi)^{2}} \int_{R^{3}} \int_{R^{3}} \frac{q(x) q(y)}{|x-y|^{2}} \mathrm{~d} x \mathrm{~d} y .
$$

The theorem was proved in [6].

Let introduce the following notation:

$$
\begin{gathered}
N A=k \int_{S^{2}} A\left(k, \theta^{\prime}, \theta\right) \mathrm{d} \theta, \\
\text { for } f=f\left(k, \theta^{\prime}, x\right), \\
D f=k \int_{S^{2}} A\left(k, \theta^{\prime}, \theta\right) f\left(k, \theta^{\prime}, x\right) \mathrm{d} \theta^{\prime}, \\
\phi_{0}(\sqrt{z}, \theta, x)=\mathrm{e}^{i \sqrt{z} \theta x}, \\
\Phi\left(\sqrt{z}, \theta^{\prime}, x\right)=\left(\Psi_{+}(\sqrt{z}, \theta, x)-\mathrm{e}^{i \sqrt{z} \theta x}\right) \Delta,
\end{gathered}
$$

where $\Delta=\prod_{j=1}^{N}\left(k+i E_{j}\right) /\left(k-i E_{j}\right)$. Define the operators $T_{ \pm}, T$ for $f \in W_{2}^{1}(R)$ as follows:

$$
\begin{array}{r}
T_{+} f=\frac{1}{2 \pi i} \lim _{\operatorname{Im} z \rightarrow 0} \int_{-\infty}^{\infty} \frac{f(s)}{s-z} \mathrm{~d} s, \operatorname{Im} z>0, \\
T_{-} f=\frac{1}{2 \pi i} \lim _{\operatorname{Im} z \rightarrow 0} \int_{-\infty}^{\infty} \frac{f(s)}{s-z} \mathrm{~d} s, \operatorname{Im} z<0, \\
T f=\frac{1}{2}\left(T_{+}+T_{-}\right) f .
\end{array}
$$

We consider the Riemann problem of finding a function $\Phi$, which is analytic in the complex plane with cut along the real axis. $\Phi$ values on the sides of the cat are denoted as $\Phi_{+}, \Phi_{-}$. Below present the results of [7].

\section{Lemma 1}

$$
\begin{aligned}
T T & =\frac{1}{4} I, T T_{+}=\frac{1}{2} T_{+}, T T_{-}=-\frac{1}{2} T_{-}, \\
T_{+} & =T+\frac{1}{2} I, T_{-}=T-\frac{1}{2} I .
\end{aligned}
$$

Theorem 4 Let $q \in \mathbf{R}, g=\left(\Phi_{+}-\Phi_{-}\right)$. Then

$$
\Phi_{ \pm}=T_{ \pm} g \text {. }
$$

The proof of the above follows from the classic results on the Riemann problem.

Lemma 2 Let $q \in \mathbf{R}, \quad g_{+}=g(\sqrt{z}, \theta, x)$, $g_{-}=g(\sqrt{z},-\theta, x)$, then

$$
\begin{aligned}
& \Psi_{+}(\sqrt{z}, \theta, x) \Delta=\left(T_{+} g_{+}+\mathrm{e}^{i \sqrt{z} \theta x}\right), \\
& \Psi_{-}(\sqrt{z}, \theta, x) \Delta=\left(T_{-} g_{-}+\mathrm{e}^{-i \sqrt{z} \theta x}\right) .
\end{aligned}
$$

The proof of the above follows from the definitions of $g, \Phi_{ \pm}, \Psi_{ \pm}$functions.

Lemma 3 Let $q \in \mathbf{R}$,

$$
\begin{aligned}
& A_{+}=A(\sqrt{z}, \theta, x), \quad A_{-}=A_{-}(\sqrt{z},-\theta, x), \text { then } \\
& A\left(k, \theta^{\prime}, \theta\right) \Delta=T_{+}\left(A_{+} \Delta-A_{-} \Delta\right) .
\end{aligned}
$$

The proof of the above follows from the definitions of $g, \Phi_{ \pm}, \Psi_{ \pm}$functions.

Lemma 4 Let $q \in \mathbf{R}$, then

$$
N A_{+} \Delta=N T_{+}(D A-\Delta) .
$$

The proof of the above follows from the definitions of $g, \Phi_{ \pm}, \Psi_{ \pm}$functions and Theorem 1.

Definition 2 Denote by $\mathbb{T} \mathbb{A}$ the set of functions $f\left(k, \theta, \theta^{\prime}\right)$ with the norm $\|f\|_{T A}=\sup _{\theta, k, \theta^{\prime}}(|T f|+|f|)<\infty$

Definition 3 Denote by $\mathbb{R}_{\left(I-D T_{-}\right)}$the set of functions $g$ such that $g=\left(I-D T_{-}\right) f$, for any $f \in \mathbb{T} \mathbb{A}$.

Lemma 5 Suppose $\|A\|_{T A}<\alpha<1$, then the operator $\left(I-D T_{-}\right)$defined on the set $\mathbb{T} \mathbb{A}$ has inverse defined on the $\mathbb{R}_{\left(I-D T_{-}\right)}$.

The proof of the above follows from the definitions of $D, T_{-}$. and conditions Lemma 5

Lemma 6 Let $q \in \mathbf{R}$ and $\left(I-T_{-} D\right)^{-1}$ is existing. Then

$$
\begin{array}{r}
g(\sqrt{z}, \theta, x)=\left(I-T_{-} D\right)^{-1} D \phi_{0}, \\
T_{-} g(\sqrt{z}, \theta, x)=T_{-}\left(I-T_{-} D\right)^{-1} D \phi_{0}, \\
\Psi_{-}=\frac{1}{\Delta} T_{-}\left(I-T_{-} D\right)^{-1} D \phi_{0}+\phi_{0} .
\end{array}
$$

The proof of the above follows from the definitions of $g, \Phi_{ \pm}, \Psi_{ \pm}$functions and Equation (4)

Lemma 7 Let $q \in \mathbf{R}$. Then

$$
q=\lim _{z \rightarrow 0} H_{0} \Psi_{-} / \Psi_{-} .
$$

The lemma can be proved substituting $\Psi_{ \pm}$in Equation (1).

Lemma 8 Let $q \in \mathbf{R}$, and $\left(I-T_{-} D\right)^{-1}$ is existing. Then

$$
q=\lim _{z \rightarrow 0} N H_{0} \Psi_{-} / N \Psi_{-},
$$




$$
q=\lim _{z \rightarrow 0} \frac{\left[\frac{1}{\Delta} N T_{-}\left(I-T_{-} D\right)^{-1} D H_{0} \phi_{0}\right]}{\left[\frac{1}{\Delta} N T_{-}\left(I-T_{-} D\right)^{-1} D \phi_{0}+N \phi_{0}\right]} .
$$

The proof of the above follows from the definitions of $N, \Psi_{ \pm}$, Lemma 6, Lemma 7.

\section{Conclusion}

This study has shown once again the outstanding properties of the scattering operator, which allow, in combination with analytical properties of the wave function, obtaining the almost explicit formulas for the potential from the scattering amplitude. Furthermore, this approach allows solving the problem of over-determination, resulting from the fact that the potential is a function of three variables, whereas the amplitude is a function of five variables. We have shown that it is sufficient to average the scattering amplitude to eliminate the two extra variables.

\section{Acknowledgements}

We thank the Ministry of Education and Science of the Republic of Kazakhstan for a grant, and the "Factor" Company of System Researches for combining our efforts in this project.

The work was performed as a part of the international project "Joint Kazakh-Indian study the influence of anthropogenic factors on atmospheric phenomena on the basis of numerical weather prediction models WRF (Weather Research and Forecasting)", commissioned by the Ministry of Education and Science of the Republic of Kazakhstan.

\section{、REFERENCES}

[1] L. D Faddeev, "Inverse Problem of Quantum Scattering Theory II,” Itogi Nauk Takh. Sov Probl Mat, Vol. 3, 1974, p. 93-180.

[2] R. G. Newton, "New Result on the Inverse Scattering Problem in Three Dimensions," Physical Review Letters, Vol. 43, No. 8, 1979, pp. 541-542.

[3] R. G. Newton, "Inverse Scattering Three Dimensions," Journal of Mathematical Physics, Vol. 21, No. 7, 1980, pp. 1698-1715. http://dx.doi.org/10.1063/1.524637

[4] E. Somersalo, et al., "Inverse Scattering Problem for the Schrodinger's Equation in Three Dimensions: Connections between Exact and Approximate Methods,” 1988.

[5] A. Y. Povzner, "On the Expansion of Arbitrary Functions in Characteristic Functions of the Operator $-\Delta u+c u$," Matematicheskii Sbornik, Vol. 32, No. 74, 1953, pp. 109156.

[6] M. Birman, "On the Spectrum of Singular BoundaryValue Problems,” (Russian) Matematicheskii Sbornik, Vol. 55, No. 97, 1961, pp. 125-174.

[7] H. Poincare, “Lecons de Mecanique Celeste,” 1910. 